\title{
Deep-Sea Bacteria Harboring Bacterial Endosymbionts in a Cytoplasm?: 3D Electron Microscopy by Serial Ultrathin Sectioning of Freeze-Substituted Specimen
}

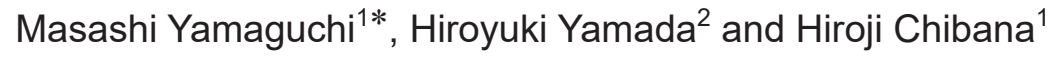 \\ ${ }^{1}$ Medical Mycology Research Center, Chiba University, 1-8-1 Inohana, Chuo-ku, Chiba 260-8673, Japan \\ ${ }^{2}$ The Research Institute of Tuberculosis, JATA (Japan Anti-Tuberculosis Association), Tokyo, Japan
}

Received March 27, 2020; accepted April 12, 2020

\begin{abstract}
Summary In 2012, we discovered a unique microorganism (Parakaryon myojinensis) that has intermediate cellular structures between prokaryotes and eukaryotes from the deep sea off the coast of Japan. Observations of ultrathin sections of deep-sea specimens with electron microscopy often revealed bacteria that contained intracellular bacteria. Here, we carried out a three-dimensional analysis of one bacterium that contained several bacteria within its cytoplasm by serial ultrathin sectioning electron microscopy of freeze-substituted specimen. We found that the host bacterium was not intact and the cell wall was broken; hence, the bacteria found inside of the host were not endosymbionts, but happen to be associated independently within cytoplasm of dead bacteria. This study emphasizes the importance of 3D analysis for understanding the interactions of microorganisms.
\end{abstract}

Keywords 3D analysis, Electron microscopy, Freeze-substitution fixation, Intracellular bacteria, Serial ultrathin sectioning.

In 2012, we discovered a unique microorganism that has intermediate cellular structures between prokaryotes and eukaryotes from the deep sea off the coast of Japan, and named it 'Parakaryon myojinensis' after the location of its discovery and its intermediate morphology (Yamaguchi et al. 2012). From our observations using ultrathin sections of freeze-substituted specimens with electron microscopy, it became apparent that there are many other strange microorganisms in the deep sea (Yamaguchi and Worman 2014, Yamaguchi 2015) including Myojin spiral bacteria (Yamaguchi et al. 2016) and Myojin amorphous bacteria (Yamaguchi et al. 2018). Among them, we often observed bacteria that contained intracellular bacteria on ultrathin sections.

Here, we carried out a three-dimensional analysis of one of the bacteria that contained several bacteria within their cytoplasm by serial ultrathin sectioning electron microscopy of the freeze-substituted specimen.

\section{Materials and methods}

Samples were collected from hydrothermal vents at the Myojin Knoll $\left(32^{\circ} 08.0^{\prime} \mathrm{N}, 139^{\circ} 51.0^{\prime} \mathrm{E}\right)$ off the coast of Japan at a depth of $1240 \mathrm{~m}$ in May 2010 (Yamaguchi et al. 2012). Small invertebrates, such as Polychaetes, and their associated microorganisms were collected and

\footnotetext{
* Corresponding author, e-mail: yama@faculty.chiba-u.jp DOI: 10.1508 /cytologia.85.209

With a Supplementary file of Movie S1.mov
}

fixed with $2.5 \%$ glutaraldehyde. They were brought to the laboratory at Chiba University, snap-frozen, freezesubstituted (Yamaguchi et al. 2011), and embedded in an epoxy resin. Serial ultrathin sections of 70-nm thickness were cut, picked up on slit grids (Yamaguchi et al. 2009, 2018), stained with uranyl acetate and lead citrate (Yamaguchi et al. 2005), and observed in a JEM-1400 electron microscope (JEOL, Tokyo, Japan) at 6000 to 40000 nominal magnifications.

3D reconstruction was performed using TrackEM2 program contained in Fiji/ImageJ software version 2.0.0-rc-43/1.50e; Java 1.6.0_65 [64-bit] (Fiji, RRID: SCR_002285, ImageJ, RRID: SCR_003070) for the eight bacterial cells analyzed in this study.

\section{Results and discussion}

Figures 1 and 2 show ultrathin sections of a bacterium that contained bacteria within its cytoplasm. The bacteria inside the cell have a cell wall, plasma membrane, ribosomes, and fibrous structure (most likely DNA fibers), and appear to be alive because of their firm ultrastructure (Figs. 1, 2). The bacteria outside the cell also have a cell wall, plasma membrane, ribosomes, and fibrous structure, and appear to be the same species as the bacteria inside the cell judging from their size and appearance (Fig. 2). The host bacterium containing the bacteria has a cell wall, plasma membrane, ribosomes, fibrous structure, and vacuoles (Figs. 1, 2). However, the vacuoles do not appear to have a membrane and seem to have 


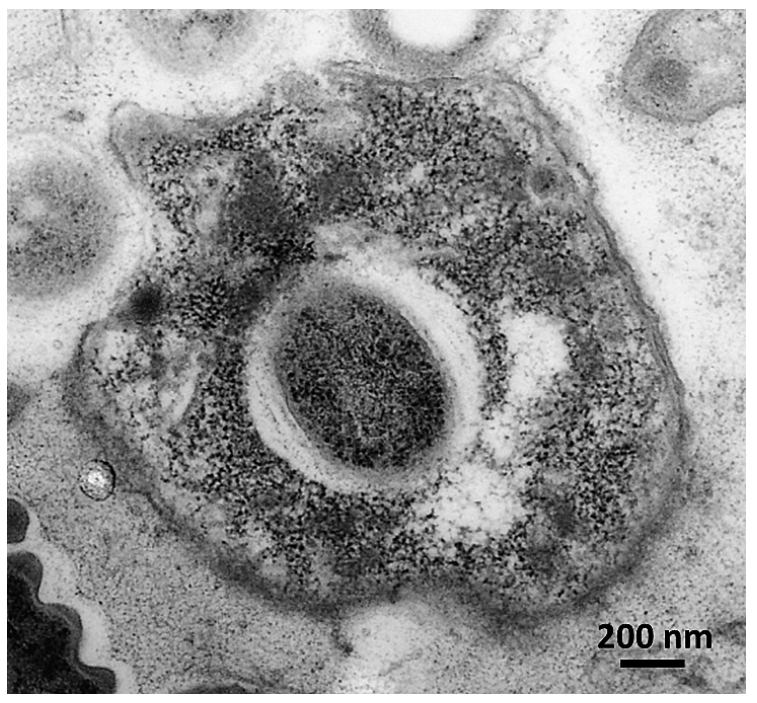

Fig. 1. Ultrathin section of a bacterium containing one bacterium.

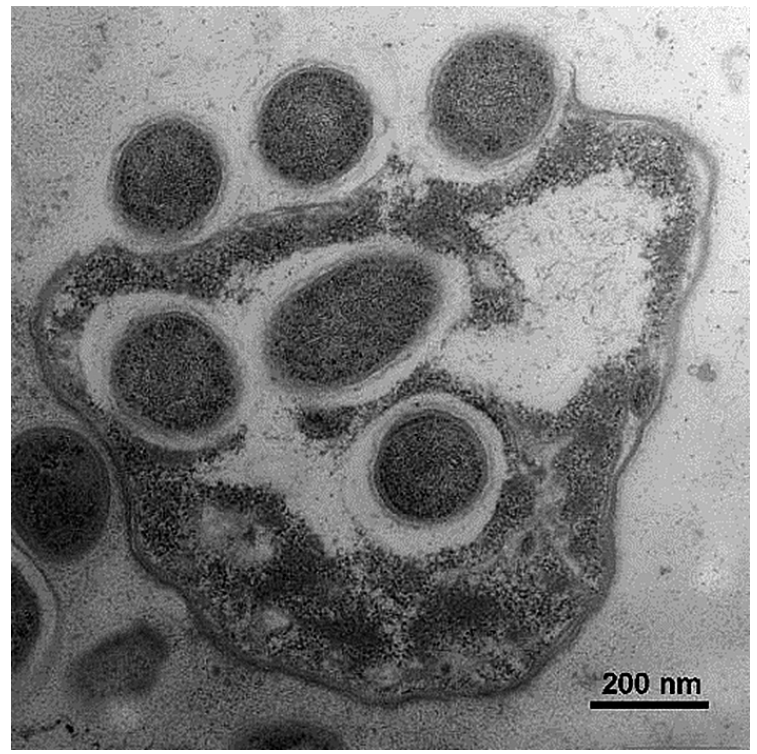

Fig. 2. Ultrathin section of a bacterium containing three bacteria.

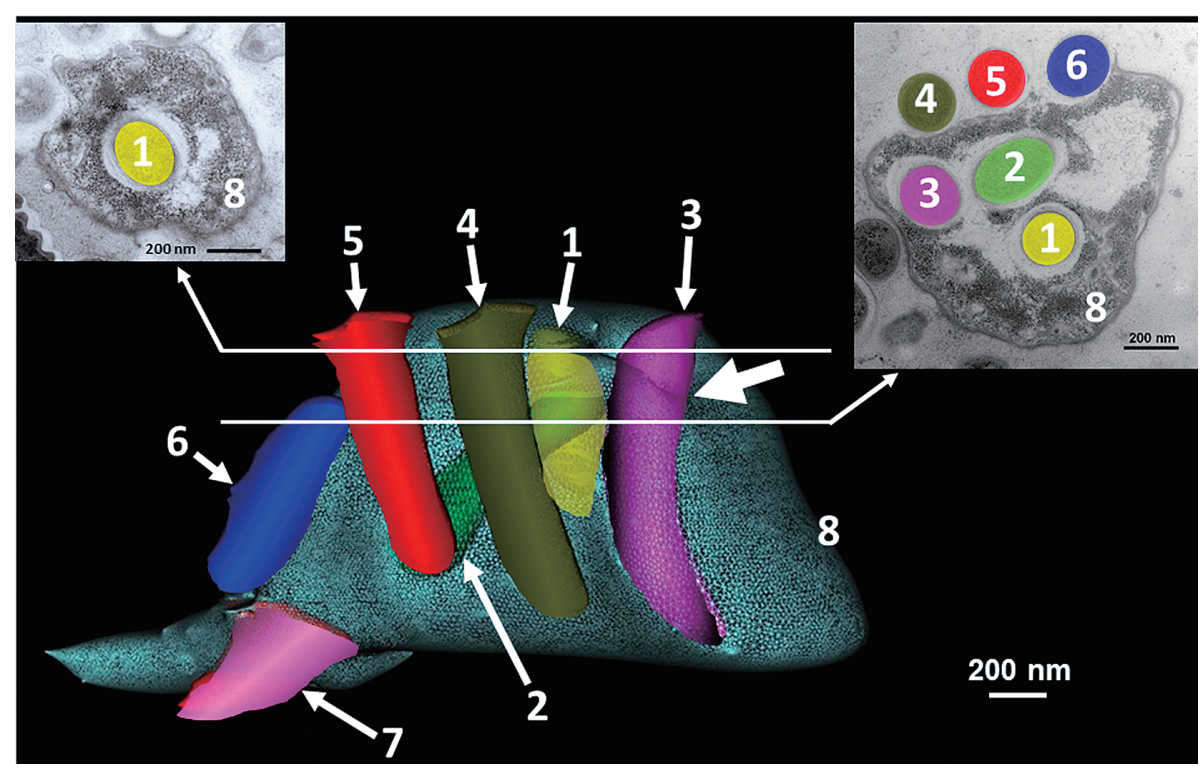

Fig. 3. Three-dimensional reconstruction image from 45 serial sections. The three bacteria (cell 1,2 , and 3 ) inside the other bacterium (cell 8) were found to be not endosymbionts but only associated with the dead bacterium whose cell wall was broken (big arrow). Fig. 1 is the 2 nd serial section, and Fig. 2 is the 15 th section of the specimen. The movie of this image is available at https://www.jstage.jst.go.jp/browse/cytologia/-char/en.

been destroyed. Fig. 3 shows a 3D image reconstructed from 45 serial ultrathin sections. Large parts of cells 1 , 2 , and 3 are located within cell 8; while cells 4, 5, 6, and 7 are located outside of cell 8 . From this image, it is apparent that the host bacterium is not intact, but its cell wall is broken, and that the cell is not alive. This indicates that the bacteria observed in the cytoplasm are not endosymbionts, but only happen to live independently in a dead bacterium.

Lane (2015) proposed that bacteria can get into the cytoplasm of other bacteria without phagocytosis in rare cases and cited Wujek's electron microscopic study (Wujek 1979) showing intracellular bacteria living inside cyanobacteria. Wujek had concluded that the intracellular bacteria are endosymbionts but had no idea what effect, if any, the bacteria have on the host nor how the bacteria got into the bacterial host.

We think that it is not possible for bacteria to get into the cytoplasm of other bacteria because of the rigid cell wall structure of the host bacteria. How, then, can the electron micrographs of bacteria containing other bacteria be explained? Wujek only observed a single ultrathin section and did not make serial ultrathin sections nor perform three-dimensional analysis. It is possible that bacteria do not actually live inside other bacteria, but are only associated with the cytoplasm of dead bacteria whose cell wall is broken. The present study of deep-sea bacteria showed that this is exactly the case based on the three- 
dimensional analysis of the specimen using serial ultrathin sections of freeze-substituted specimens. The association of the bacteria with dead bacteria could also have been artificially caused by the centrifugation steps used for the preparation of specimens for electron microscopy.

Whether bacteria can enter inside other bacteria without phagocytosis is a critical issue in considering the origin of eukaryote cells. P. myojinensis, a unique microorganism that we found in the deep sea, contained endosymbionts that resemble bacteria (Yamaguchi et al. 2012). Lane, in some kind of a recapitulation of the evolution of life, suggested that $P$. myojinensis is a prokaryote that has acquired endosymbionts without phagocytosis and is changing into a cell that resembles a eukaryote (Lane 2015). We think that $P$. myojinensis had acquired bacterial endosymbionts by phagocytosis a long time ago when it had no cell walls and then later formed cell walls. Therefore, the vacuoles which are bounded by membranes and contained the endosymbionts may be phagosomes and are not artifacts of shrinkage, particularly because freeze-substitution fixation keeps the cell structures in their natural state.

We, therefore, suggest the reexamination of the occurrence of intracellular bacteria in other bacteria using serial ultrathin sections and three-dimensional reconstruction of freeze-substituted samples to verify whether bacteria observed within other bacteria are actually endosymbionts or merely associated with the cytoplasm of dead bacteria.

\section{Acknowledgements}

We thank the crew of the research vessel Natsushima and the staff of Japan Marine Science and Technology for their support of the NT10-08 cruise.

\section{References}

Lane, N. 2015. Epilogue: From the Deep. In: The Vital Question: Energy, Evolution, and the Origins of Complex Life. W. W. Norton and Company, New York. pp. 281-290.

Wujek, D. E. 1979. Intracellular bacteria in the blue-green alga Pleurocapsa minor. Trans. Am. Microsc. Soc. 98: 143-145.

Yamaguchi, M. 2015. An electron microscopic study of microorganisms: From influenza virus to deep-sea microorganisms. JSM Mycotoxins 65: 81-99.

Yamaguchi, M., Mori, Y., Kozuka, Y., Okada, H., Uematsu, K., Tame, A., Furukawa, H., Maruyama, T., Worman, C. O. and Yokoyama, K. 2012. Prokaryote or eukaryote? A unique microorganism from the deep-sea. J. Electron Microsc. 61: 423-431.

Yamaguchi, M., Namiki, Y., Okada, H., Uematsu, K., Tame, A., Maruyama, T. and Kozuka, Y. 2011. Improved preservation of fine structure of deep-sea microorganisms by freeze-substitution after glutaraldehyde fixation. J. Electron Microsc. 60: 283-287.

Yamaguchi, M., Okada, H. and Namiki, Y. 2009. Smart specimen preparation for freeze substitution and serial ultrathin sectioning of yeast cells. J. Electron Microsc. 58: 261-266.

Yamaguchi, M., Shimizu, M., Yamaguchi, T., Ohkusu, M. and Kawamoto, S. 2005. Repeated use of uranyl acetate solution for section staining in transmission electron microscopy. Plant Morphol. 17: 57-59.

Yamaguchi, M. and Worman, C. O. 2014. Deep-sea microorganisms and the origin of the eukaryotic cell. Jpn. J. Protozool. 47: 29-48.

Yamaguchi, M., Yamada, H., Higuchi, K., Yamamoto, Y., Arai, S., Murata, K., Mori, Y., Furukawa, H., Uddin, M. S. and Chibana, H. 2016. High-voltage electron microscopy tomography and structome analysis of unique spiral bacteria from the deep sea. Microscopy 65: 363-369.

Yamaguchi, M., Yamada, H., Uematsu, K., Horinouchi, Y. and Chibana, H. 2018. Electron microscopy and structome analysis of unique amorphous bacteria from the deep sea in Japan. Cytologia 83: 337-342. 